\title{
A NONLINEAR INTEGRAL EQUATION AND AN ITERATIVE ALGORITHM FOR AN INVERSE SOURCE PROBLEM
}

\author{
RAINER KRESS AND WILLIAM RUNDELL \\ Communicated by Paul Martin
}

\begin{abstract}
We consider the inverse problem of recovering the shape of an extended source of known homogeneous strength within a conducting medium from one voltage and current measurement on the accessible boundary of the medium and present an iterative solution method via boundary integral equations. The main idea of our approach is to equivalently reformulate the inverse source problem as an inverse boundary value problem with a non-local Robin condition on the boundary of the source domain. Following our approach in [12] for an inverse Dirichlet problem, from Green's representation formula we obtain a nonlinear integral equation for the unknown boundary curve which can be solved by regularized Newton iterations. We present the foundations of the inverse algorithm and illustrate its feasibility by some numerical examples.
\end{abstract}

1. Introduction. Electrostatic imaging models the detection of inclusions or sources within a conducting medium from voltage and currents measurements on its boundary and leads to inverse problems for the Laplace equation. In this paper, we will examine an inverse source problem with an extended source spread over a subdomain of the conducting medium. For this, we assume that $\Omega$ is a doubly connected bounded domain in $\mathbb{R}^{2}$ with boundary $\partial \Omega$ that consists of two disjoint $C^{2}$ smooth closed Jordan curves $\Gamma_{m}$ and $\Gamma_{s}$ such that $\partial \Omega:=\Gamma_{m} \cup \Gamma_{s}$ and $\Gamma_{s}$ is contained in the interior of $\Gamma_{m}$. Here, $\Gamma_{s}$ stands for the boundary of an extended source to be determined, and $\Gamma_{m}$ stands for

2010 AMS Mathematics subject classification. Primary 31A25, 45Q05, 49N45.

The work of the second author was partly supported by NSF grant DMS1319052. Funding for the visit of the first author to Texas A\&M University that made the completion of this paper possible was also from the NSF.

Received by the editors on September 9, 2014, and in revised form on December 13, 2014. 
the measurement surface. We denote by $\Omega_{s}$ and $\Omega_{m}$ the bounded domains with boundaries $\Gamma_{s}$ and $\Gamma_{m}$, respectively. By $\nu$, we denote the unit normal vector to $\Gamma_{s}$ and to $\Gamma_{m}$ that is directed into the exterior of $\Omega_{s}$ and the exterior of $\Omega_{m}$, respectively.

Our topic is the inverse problem of reconstructing the inclusion $\Omega_{s}$ from the Cauchy data on $\Gamma_{m}$ of a solution $u \in H^{1}\left(\Omega_{m}\right)$ of the source equation

$$
\Delta u=\chi_{s} \text { in } \Omega_{m},
$$

where $\chi_{s}$ denotes the characteristic function of $\Omega_{s}$. We note that, in principle, we need only consider the problem with Dirichlet data $f=0$. For a given Cauchy pair $(f, g)$ on $\Gamma_{m}$ of a solution $u$ of (1.1), the function $v=u-u_{f}$ where $u_{f} \in H^{1}\left(\Omega_{m}\right)$ is the unique harmonic function with $u_{f}=f$ on $\partial \Omega_{m}$ again satisfies (1.1) but with zero Dirichlet data. This shows that no further information can be gained by using different Dirichlet data since the harmonic function $u_{f}$ does not carry any information on $\Gamma_{s}$. A similar argument shows that we also could restrict ourselves to the case of constant Neumann data $g=\left|\Omega_{s}\right| /\left|\Gamma_{m}\right|$. However, for convenience, we present our inverse algorithm for the general case. Concerning uniqueness, we note that $\Gamma_{s}$ is uniquely determined by one Cauchy pair, provided it is star-like with respect to the center of gravity of $\Omega_{s}$ and the radial distance function describing $\Gamma_{s}$ is Lipschitz continuous (see [5, Theorem 4.1.1]).

We propose a new iterative scheme for the numerical solution of the above inverse source problem. The main idea of our approach is to equivalently reformulate the Dirichlet problem for the source equation (1.1) in $\Omega_{m}$ as a boundary value problem for the Laplace equation in $\Omega$ with a Dirichlet boundary condition on $\Gamma_{m}$ and a non-local Robin condition on $\Gamma_{s}$ in terms of the Dirichlet-to-Neumann operator for harmonic functions in $\Omega_{s}$. Then, following our approach in [12] for an inverse Dirichlet problem, from Green's representation formula applied to the solution of the combined Dirichlet and Robin problem we obtain a nonlinear integral equation for the unknown boundary curve $\Gamma_{s}$ which can be solved by regularized Newton iterations.

As alternative methods for the same problem we mention the approach by Hettlich and Rundell [4] who suggested Newton iterations for the boundary-to-data map associated with the source problem. More recently, Hanke and Rundell [3] proposed another Newton type itera- 
tion in the framework of the reciprocity gap principle using trigonometric harmonics as test functions. Relations of our approach with these two methods will be discussed at the end of Section 3 .

The plan of the paper is as follows. Section 2 provides two boundary integral equation methods for solving the direct source problem and introduces notation. We then proceed in Section 3 with the presentation of our reconstruction algorithm for the shape $\Gamma_{s}$ including a short discussion of some relations to the regularized Newton iterations for the boundary-to-data map as proposed in [4]. In the final Section 4 we will provide numerical examples illustrating the feasibility of the inverse algorithm.

The method described in [12] for the inverse problem with a Dirichlet condition on $\Gamma_{s}$ and in [6] for a Neumann condition on $\Gamma_{s}$ has been recently also applied to a local Robin or impedance condition by Cakoni et al. [1].

In principle, our proposed method can be also used for the case where the source domain $\Omega_{s}$ consists of a finite number of disjoint subsets provided a priori information on the number, the location and the size of the subsets is available. This a priori information, for example, could be obtained by a rational approximation algorithm as developed recently by Hanke and Rundell [3].

Finally, we also note that our method allows a straightforward extension to the Helmholtz equation with a source term.

2. The direct problem. We begin by studying the Dirichlet problem for (1.1), subject to the boundary condition

$$
u=f \quad \text { on } \partial \Omega_{m}
$$

for a given $f \in H^{1 / 2}\left(\Omega_{m}\right)$ as the corresponding direct problem. In particular, we present two different approaches for solving this boundary value problem by boundary integral equations. Although the first approach we are describing will not be useful for the solution of the inverse problem we found it helpful to have two independent methods available for the direct problem. In the course of this section the reader also will get familiar with the integral operators that will be needed for our inverse algorithm. 
For the first method, in terms of the fundamental solution

$$
\Phi(x, y)=\frac{1}{2 \pi} \ln \frac{1}{|x-y|}, \quad x \neq y
$$

we introduce the single- and double-layer potential operators

$$
S_{j k}: H^{-1 / 2}\left(\Gamma_{j}\right) \longrightarrow H^{1 / 2}\left(\Gamma_{k}\right)
$$

and

$$
K_{j k}: H^{1 / 2}\left(\Gamma_{j}\right) \longrightarrow H^{1 / 2}\left(\Gamma_{k}\right)
$$

defined by

$$
\left(S_{j k} \varphi\right)(x):=2 \int_{\Gamma_{j}} \Phi(x, y) \varphi(y) d s(y), \quad x \in \Gamma_{k},
$$

and

$$
\left(K_{j k} \varphi\right)(x):=2 \int_{\Gamma_{j}} \frac{\partial \Phi(x, y)}{\partial \nu(y)} \varphi(y) d s(y), \quad x \in \Gamma_{k}
$$

for $j, k=m, s$. Without loss of generality, we assume that there exists a point $x_{m}$ in $\Omega_{m}$ such that $\left|x-x_{m}\right| \neq 1$ for all $x \in \Gamma_{m}$ and a point $x_{s}$ in $\Omega_{s}$ such that $\left|x-x_{s}\right| \neq 1$ for all $x \in \Gamma_{s}$. Then Theorem 3.16 in [9] (see also [10, Theorem 7.38]) guarantees that the single-layer operators $S_{j j}: H^{-1 / 2}\left(\Gamma_{j}\right) \rightarrow H^{1 / 2}\left(\Gamma_{j}\right)$ are injective for $j=m, s$. In the sequel, we will refer to this as assumption $\mathrm{G}$. Note that, by a change of variables, any domains $\Omega_{m}$ and $\Omega_{s}$ always can be scaled to a setting where the condition $\mathrm{G}$ is satisfied.

Here, for simplicity, we have chosen just to work with the singlelayer operators and point out that, alternatively, in order to obtain injectivity, we could modify them by adding an additional term as in [10, Theorem 7.41]. We also note that in $\mathbb{R}^{3}$, the condition $G$ is not required for the injectivity of the single-layer operators.

We introduce a special solution $u_{0}$ of the Poisson equation

$$
\Delta u_{0}=1
$$

in $\Omega_{s}$ by

$$
u_{0}(x):=\frac{1}{4}|x|^{2}, \quad x \in \Omega_{s},
$$


and note that for our purposes (2.5) can be replaced by any other explicit expression for a solution to (2.4). In our first approach we search the solution of the Dirichlet problems (1.1) and (2.1) in the form

$$
u= \begin{cases}v & \text { in } \Omega, \\ u_{0}+v & \text { in } \Omega_{s},\end{cases}
$$

with a potential

$$
\begin{aligned}
v(x)= & \int_{\Gamma_{s}}\left\{\Phi(x, y) \psi(y)+\frac{\partial \Phi(x, y)}{\partial \nu(y)} \eta(y)\right\} d s(y) \\
& +\int_{\Gamma_{m}} \frac{\partial \Phi(x, y)}{\partial \nu(y)} \varphi(y) d s(y), \quad x \in \Omega \cup \Omega_{s} .
\end{aligned}
$$

By the jump relations, assuming that $\varphi \in H^{1 / 2}\left(\Gamma_{m}\right)$ and choosing

$$
\psi=-\left.\frac{\partial u_{0}}{\partial \nu}\right|_{\Gamma_{s}} \text { and } \eta=\left.u_{0}\right|_{\Gamma_{s}}
$$

ensures that $u$ is an $H^{1}\left(\Omega_{m}\right)$ solution of (1.1). The Dirichlet boundary condition (2.1) is satisfied provided $\varphi$ solves the integral equation

$$
-\varphi+K_{m m} \varphi=2 f-S_{s m} \psi-K_{s m} \eta \text {. }
$$

Clearly, by the classical results on the double-layer integral equation of the second kind for the interior Dirichlet problem (see [10]), the equation (2.8) possesses a unique solution $\varphi \in H^{1 / 2}\left(\Gamma_{m}\right)$. For its approximate solution, an efficient Nyström method based on trigonometric approximations as described, for example, in [10] can be employed.

Note, that this approach is not suited for solving the inverse problem since in this case in addition to $\Gamma_{s}$ and $\varphi$ also $\psi$ and $\eta$ would be unknown.

For the second approach for solving the direct problem in addition to the single- and double-layer boundary operators we also need the corresponding normal derivative operators

$$
K_{j k}^{\prime}: H^{-1 / 2}\left(\Gamma_{j}\right) \longrightarrow H^{-1 / 2}\left(\Gamma_{k}\right)
$$

and

$$
T_{j k}: H^{1 / 2}\left(\Gamma_{j}\right) \longrightarrow H^{-1 / 2}\left(\Gamma_{k}\right)
$$


defined by

$$
\left(K_{j k}^{\prime} \varphi\right)(x):=2 \int_{\Gamma_{j}} \frac{\partial \Phi(x, y)}{\partial \nu(x)} \varphi(y) d s(y), \quad x \in \Gamma_{k}
$$

and

$$
\begin{gathered}
\left(T_{j k} \varphi\right)(x):=2 \frac{\partial}{\partial \nu(x)} \int_{\Gamma_{j}} \frac{\partial \Phi(x, y)}{\partial \nu(y)} \varphi(y) d s(y), \\
x \in \Gamma_{k},
\end{gathered}
$$

for $j, k=m, s$. Further we introduce the Dirichlet-to-Neumann operator $A: H^{1 / 2}\left(\Gamma_{s}\right) \rightarrow H^{-1 / 2}\left(\Gamma_{s}\right)$ for the domain $\Omega_{s}$ mapping the Dirichlet trace on $\Gamma_{s}$ of harmonic functions in $H^{1}\left(\Omega_{s}\right)$ onto the Neumann trace on $\Gamma_{s}$. From the existence analysis for the interior Dirichlet problem via the double-layer integral equation, we have the representation

$$
A=T_{s s}\left(K_{s s}-I\right)^{-1} \text {. }
$$

Alternatively, using the single-layer integral equation, we also have

$$
A=\left(K_{s s}^{\prime}+I\right) S_{s s}^{-1}
$$

provided the geometric assumption G ensuring injectivity of $S_{s s}$ is imposed. (Details on the representations (2.11) and (2.12) are described, for example, in [10].)

For a solution $u \in H^{1}\left(\Omega_{m}\right)$ of (1.1), the function $u-u_{0}$ in $\Omega_{s}$ (recall the definition (2.5)) is harmonic, and we have

$$
\left.\frac{\partial\left(u-u_{0}\right)}{\partial \nu}\right|_{\Gamma_{s}}=\left.A\left(u-u_{0}\right)\right|_{\Gamma_{s}} .
$$

Therefore, in view of the continuity of $u$ and its normal derivative across $\Gamma_{s}$, the source problem (1.1) and (2.1) can be separated into two parts. First we solve for a harmonic function $u$ in $\Omega$ with Dirichlet condition (2.1) on $\Gamma_{m}$ and the non-local Robin condition

$$
\frac{\partial u}{\partial \nu}-A u=\frac{\partial u_{0}}{\partial \nu}-\left.A u_{0}\right|_{\Gamma_{s}} \quad \text { on } \Gamma_{s}
$$

Then, knowing $\left.u\right|_{\Gamma_{s}}$, we solve for a harmonic function $v$ in $\Omega_{s}$ with Dirichlet values $v=u-u_{0}$ on $\Gamma_{s}$ and set $u:=u_{0}+v$ in $\Omega_{s}$. (Note that if we are only interested in the Neumann trace of $u$ on $\Gamma_{m}$ the second 
part can be omitted.) Existence of the solution to the mixed Dirichlet and Robin problems (2.1) and (2.13) is evident as restriction of the solution to the original source problems (1.1) and (2.1) and uniqueness follows from Green's integral theorem and the positive definiteness of the Dirichlet-to-Neumann map $A$.

There are various possibilities for solving the mixed Dirichlet and Robin problems (2.1) and (2.13). By the jump relations, a single-layer approach on both boundary components

$$
\begin{aligned}
u(x)= & \int_{\Gamma_{m}} \varphi_{m}(y) \Phi(x, y) d s(y) \\
& \quad+\int_{\Gamma_{s}} \varphi_{s}(y) \Phi(x, y) d s(y), \quad x \in \Omega,
\end{aligned}
$$

satisfies both (2.1) and (2.13), provided that $\varphi_{m} \in H^{-1 / 2}\left(\Gamma_{m}\right)$ and $\varphi_{s} \in H^{-1 / 2}\left(\Gamma_{s}\right)$ solve the system of integral equations

$$
\begin{gathered}
S_{m m} \varphi_{m}+S_{s m} \varphi_{s}=f \\
K_{m s}^{\prime} \varphi_{m}-A S_{m s} \varphi_{m}-\varphi_{s}+K_{s s}^{\prime} \varphi_{s}-A S_{s s} \varphi_{s}=\frac{\partial u_{0}}{\partial \nu}-\left.A u_{0}\right|_{\Gamma_{s}}
\end{gathered}
$$

Theorem 2.1. Provided the geometric assumption $G$ is satisfied, the system of boundary integral equations (2.15)-(2.16) is uniquely solvable.

Proof. Assume that $\varphi_{m}$ and $\varphi_{s}$ solve the homogeneous form of (2.15)-(2.16), and define $u$ by (2.14) in all of $\mathbb{R}^{2}$. Then, in view of the uniqueness for the mixed Dirichlet and Robin problem in $\Omega$ we have that $u=0$ in $\Omega$. From $u=0$ on $\Gamma_{s}$ and the uniqueness for the Dirichlet problem in $\Omega_{s}$ it follows that $u=0$ in $\Omega_{s}$, and consequently $\varphi_{s}=0$ by the jump relations. Now, finally we have that $S_{m m} \varphi_{m}=0$, and injectivity of $S_{m m}$ ensures that $\varphi_{m}=0$. Thus, we have proved injectivity for (2.15)-(2.16).

Using the identity

$$
T_{m m} S_{m m}=K_{m m}^{\prime 2}-I
$$

(see [10]) the system (2.15)-(2.16) can be equivalently transformed into a system with a compact perturbation of the identity. Hence, by the Riesz theory, injectivity implies existence of a solution. 
Again, the boundary integral equations (2.15)-(2.16) can be efficiently solved via a combination of Nyström and collocation methods based on trigonometric polynomial approximations that take proper care of the logarithmic singularity in the single-layer operators. When the representation (2.11) of the Dirichlet-to-Neumann map $A$ is used, the implementation of the hypersingular operator $T_{s s}$ can be reduced to the implementation of $S_{s s}$ by employing Maue's formula

$$
T_{s s} \varphi=\frac{d}{d s} S_{s s} \frac{d \varphi}{d s}
$$

and trigonometric differentiation as described in [10, subsection 13.5], see also $[\mathbf{1 1}]$.

For creating the synthetic data to be used in the inverse algorithm, we found it quite convenient to have two independent methods available for verifying the correctness of our numerical implementation. From the general error analysis in [10], it can be concluded that for analytic boundaries and analytic Dirichlet data both methods are exponentially convergent.

Table 1 gives some approximate values for the parameterized normal derivative $\widetilde{g}=g \circ z_{m}$ for the apple-shaped $\Gamma_{s}$ given by (4.4) and the circle $\Gamma_{m}$ given by (4.6) in Section 4. The Dirichlet data are $f=0$. The exponential convergence is clearly exhibited. The results in Table 1 are obtained via the integral equation (2.8). For $n=64$, the method based on the non-local Robin condition via the system of integral equations (2.15)-(2.16) gives the same results up to 12 decimals. In both cases, the normal derivative of the double-layer potential on $\Gamma_{m}$ is discretized via Maue's formula.

TABLE 1. Numerical solution for direct problem.

\begin{tabular}{|r|c|c|c|c|}
\hline$n$ & $\widetilde{g}(0)$ & $\widetilde{g}(\pi / 2)$ & $\widetilde{g}(\pi)$ & $\widetilde{g}(3 \pi / 2)$ \\
\hline & & & & \\
8 & 0.113024245219 & 0.139099807477 & 0.144891779304 & 0.126847715069 \\
16 & 0.113105431204 & 0.138908670358 & 0.144111296858 & 0.125685584383 \\
32 & 0.113083117312 & 0.138918100239 & 0.144118988822 & 0.125692471507 \\
64 & 0.113083118465 & 0.138918099791 & 0.144118988474 & 0.125692471222 \\
\hline
\end{tabular}

We conclude this section by noting that the above methods have immediate extensions both to the source equation with other right 
hand sides (instead of the characteristic function) and to the Helmholtz equation.

3. The nonlinear integral equation. Recall that the inverse problem is to recover $\Gamma_{s}$ from the Cauchy data

$$
f=\left.u\right|_{\Gamma_{m}} \quad \text { and } \quad g:=\left.\frac{\partial u}{\partial \nu}\right|_{\Gamma_{m}}
$$

of a solution $u$ to (1.1). Following the approach for the solution of an inverse Dirichlet problem as suggested in [12], from Green's representation formula applied to $u$ in the domain $\Omega$ we obtain the following equivalent integral equation.

Theorem 3.1. The inverse problem is equivalent to solving the nonlinear integral equation

$$
\begin{gathered}
-\frac{1}{2}\left[K_{s m}-S_{s m} A\right]\left(K_{m s} f-S_{m s} g+\left.S_{s s} \frac{\partial u_{0}}{\partial \nu}\right|_{\Gamma_{s}}\right. \\
\left.-\left.K_{s s} u_{0}\right|_{\Gamma_{s}}-\left.u_{0}\right|_{\Gamma_{s}}\right) \\
=f+K_{m m} f-S_{m m} g+S_{s m}\left(\left.\frac{\partial u_{0}}{\partial \nu}\right|_{\Gamma_{s}}-\left.A u_{0}\right|_{\Gamma_{s}}\right)
\end{gathered}
$$

for the unknown $\Gamma_{s}$.

Proof. For a harmonic function $u \in H^{1}(\Omega)$ with Cauchy data $(f, g)$ on $\Gamma_{m}$ that satisfies the non-local Robin condition (2.13) we set

$$
\varphi:=\left.u\right|_{\Gamma_{s}}
$$

Then by Green's formula we have that

$$
\begin{aligned}
u(x)= & \int_{\Gamma_{s}}\left\{\frac{\partial \Phi(x, y)}{\partial \nu(y)} \varphi(y)\right. \\
& \left.-\Phi(x, y)\left(\left.\frac{\partial u_{0}}{\partial \nu}\right|_{\Gamma_{s}}+A \varphi-\left.A u_{0}\right|_{\Gamma_{s}}\right)(y)\right\} d s(y) \\
& +\int_{\Gamma_{m}}\left\{\Phi(x, y) g(y)-\frac{\partial \Phi(x, y)}{\partial \nu(y)} f(y)\right\} d s(y), \quad x \in \Omega
\end{aligned}
$$


Letting $x$ tend to $\Gamma_{m}$ and $\Gamma_{s}$ from inside $\Omega$, we obtain the integral equations

$$
\begin{aligned}
K_{s m} \varphi-S_{s m} A \varphi= & f+K_{m m} f-S_{m m} g \\
& +S_{s m}\left(\left.\frac{\partial u_{0}}{\partial \nu}\right|_{\Gamma_{s}}-\left.A u_{0}\right|_{\Gamma_{s}}\right)
\end{aligned}
$$

and

$$
-\varphi+K_{s s} \varphi-S_{s s} A \varphi=K_{m s} f-S_{m s} g+S_{s s}\left(\left.\frac{\partial u_{0}}{\partial \nu}\right|_{\Gamma_{s}}-\left.A u_{0}\right|_{\Gamma_{s}}\right)
$$

for the unknowns $\varphi$ and $\Gamma_{s}$. Using (2.11) and

$$
S_{s s} T_{s s}=K_{s s}^{2}-I \text {, }
$$

we have that

$$
S_{s s} A=K_{s s}+I .
$$

Hence, (3.4) is equivalent to

$$
-2 \varphi=K_{m s} f-S_{m s} g+\left.S_{s s} \frac{\partial u_{0}}{\partial \nu}\right|_{\Gamma_{s}}-\left.K_{s s} u_{0}\right|_{\Gamma_{s}}-\left.u_{0}\right|_{\Gamma_{s}} .
$$

Inserting this into (3.3), we obtain (3.1).

Conversely, assume $\Gamma_{s}$ solves (3.1). Then we define $\varphi$ via (3.6) and have that $\varphi$ also satisfies (3.4). We further define $u$ in $\mathbb{R}^{2} \backslash \partial \Omega$ by the right hand side of (3.2). Then, (3.3) and (3.4) imply that $u=0$ when approaching $\Gamma_{m}$ and $\Gamma_{s}$ from outside $\Omega$. To conclude $u=0$ in $\mathbb{R}^{2} \backslash \bar{\Omega}$ via uniqueness for the exterior and interior Dirichlet problem we need to proceed analogous to the proof of Theorem 3.16 in [9] using our geometric assumption G. When approaching $\partial \Omega$ from inside $\Omega$, the jump relations imply that

$$
u=f \quad \text { and } \quad \frac{\partial u}{\partial \nu}=g \quad \text { on } \Gamma_{m}
$$

and

$$
u=\varphi \quad \text { and } \quad \frac{\partial u}{\partial \nu}=\frac{\partial u_{0}}{\partial \nu}+A \varphi-\left.A u_{0}\right|_{\Gamma_{s}} \text { on } \Gamma_{s} .
$$

As worked out in Section 2, the latter ensures that $u$ can be extended from $\Omega$ into $\Omega_{m}$ as an $H^{1}$ solution of (1.1). Hence, $\Gamma_{s}$ solves the inverse problem for the given Cauchy pair $(f, g)$. 
The integral equation (3.1) now can be approximately solved via linearization with respect to $\Gamma_{s}$ and iteration, that is, by regularized Newton iterations. The Dirichlet trace $\varphi$ is only of secondary interest in connection with the inverse problem and therefore can be viewed as a slip variable. The fortunate fact that it can be eliminated in a straightforward manner due to the transformation of (3.4) into (3.6) is not typical for the approach to derive equivalent nonlinear integral equations via Green's theorems for this type of inverse boundary value problem. In general, the method proposed in [12] uses two equations for the unknown shape $\Gamma_{s}$ and for a density function depending on the type of boundary condition on $\Gamma_{s}$ and linearizes both equations with respect to both unknowns and then iterates.

Nevertheless, also in the present case, in the spirit of the method proposed first by Johansson and Sleeman [7], for an inverse scattering problem, it is tempting to try an alternative method. In this iteration scheme, given an approximation for $\Gamma_{s}$, we determine the density $\varphi$ from the transformed equation (3.6). Then keeping $\varphi$ fixed, we update $\Gamma_{s}$ by linearizing (3.3) with respect to $\Gamma_{s}$. However, our numerical experiments with this approach did not result in a convergent scheme. Knowing that the approach from [7] is sensitive to whether the arc length is integrated into the slip variable $\varphi$ (see also a corresponding discussion in [12]) we assume that this lack of convergence is due to the fact that we choose the slip variable $\varphi$ such that we avoided the occurrence of the arc length in our parameterized integral operators in order to obtain simpler expressions for the linearizations.

For the foundation of these linearizations and the Newton type iterations we need the Fréchet derivatives of the involved single- and double-layer operators with respect to $\Gamma_{s}$. To this end, without much loss of generality, we assume that the $C^{2}$-boundaries $\Gamma_{j}$ for $j=m, s$ have parametric representations with counter clockwise orientation

$$
\Gamma_{j}=\left\{z_{j}(t): t \in[0,2 \pi]\right\}
$$

with $2 \pi$ periodic $C^{2}$ smooth functions $z_{j}: \mathbb{R} \rightarrow \mathbb{R}^{2}$ such that $z_{j}$ is injective on $[0,2 \pi)$. In view of $(2.2)$ and $(2.3)$, we introduce parameterized single- and double-layer operators

$$
\widetilde{S}_{j k}: H_{\mathrm{per}}^{-1 / 2}[0,2 \pi] \longrightarrow H_{\mathrm{per}}^{1 / 2}[0,2 \pi]
$$


and

$$
\widetilde{K}_{j k}: H_{\mathrm{per}}^{1 / 2}[0,2 \pi] \longrightarrow H_{\mathrm{per}}^{1 / 2}[0,2 \pi]
$$

by

$$
\widetilde{S}_{j k}(\psi)(t):=\frac{1}{\pi} \int_{0}^{2 \pi} \ln \frac{1}{\left|z_{k}(t)-z_{j}(\tau)\right|} \psi(\tau) d \tau, \quad t \in[0,2 \pi]
$$

and

$$
\begin{gathered}
\widetilde{K}_{j k}(\psi)(t):=\frac{1}{\pi} \int_{0}^{2 \pi} \frac{\left[z_{j}^{\prime}(\tau)\right]^{\perp} \cdot\left[z_{k}(t)-z_{j}(\tau)\right]}{\left|z_{k}(t)-z_{j}(\tau)\right|^{2}} \psi(\tau) d \tau, \\
t \in[0,2 \pi],
\end{gathered}
$$

for $j, k=m, s$. Here, we write $a^{\perp}=\left(a_{2},-a_{1}\right)$ for any vector $a=$ $\left(a_{1}, a_{2}\right)$, that is, $a^{\perp}$ is obtained by rotating $a$ clockwise by 90 degrees. In view of the representation (2.11) of the Dirichlet-to-Neumann map and Maue's formula (2.17), we further introduce the parameterized Dirichlet-to-Neumann operator $\widetilde{A}: H_{\text {per }}^{1 / 2}[0,2 \pi] \rightarrow H_{\text {per }}^{-1 / 2}[0,2 \pi]$ by

$$
\widetilde{A} \psi:=\frac{d}{d t} \widetilde{S}_{s s} \frac{d}{d t}\left(\widetilde{K}_{s s}-I\right)^{-1} \psi
$$

Then the parameterized version of (3.1) becomes

$$
\begin{aligned}
& -\frac{1}{2}\left[\widetilde{K}_{s m}-\widetilde{S}_{s m} \widetilde{A}\right]\left(\widetilde{K}_{m s} \widetilde{f}-\widetilde{S}_{m s} \widetilde{g}+\widetilde{S}_{s s} \widetilde{v}_{0}-\widetilde{K}_{s s} \widetilde{u}_{0}-\widetilde{u}_{0}\right) \\
& =\widetilde{f}+\widetilde{K}_{m m} \widetilde{f}-\widetilde{S}_{m m} \widetilde{g}+\widetilde{S}_{s m}\left(\widetilde{v}_{0}-\widetilde{A} \widetilde{u}_{0}\right)
\end{aligned}
$$

which needs to be solved for $z_{s}$, and where we set

$$
\tilde{f}=f \circ z_{m}, \quad \widetilde{g}=\left|z_{m}^{\prime}\right| g \circ z_{m}
$$

and

$$
\begin{aligned}
& \widetilde{u}_{0}=u_{0} \circ z_{s}=\frac{1}{4}\left|z_{s}\right|^{2} \\
& \widetilde{v}_{0}=\left|z_{s}^{\prime}\right| \frac{\partial u_{0}}{\partial \nu} \circ z_{s}=\frac{1}{2}\left[z_{s}^{\prime}\right]^{\perp} \cdot z_{s} .
\end{aligned}
$$

Collecting all the terms in (3.11) that depend on $z_{s}$, we define the 
operator $F: H_{\text {per }}^{2}[0,2 \pi] \rightarrow L^{2}[0,2 \pi]$ by

$$
\begin{aligned}
F\left(x_{s}\right):= & -\frac{1}{2}\left[\widetilde{K}_{s m}-\widetilde{S}_{s m} \widetilde{A}\right]\left(\widetilde{K}_{m s} \widetilde{f}-\widetilde{S}_{m s} \widetilde{g}+\widetilde{S}_{s s} \widetilde{v}_{0}-\widetilde{K}_{s s} \widetilde{u}_{0}-\widetilde{u}_{0}\right) \\
& -\widetilde{S}_{s m}\left(\widetilde{v}_{0}-\widetilde{A} \widetilde{u}_{0}\right)
\end{aligned}
$$

and a right hand side $h \in L^{2}[0,2 \pi]$ by

$$
h:=\tilde{f}+\widetilde{K}_{m m} \tilde{f}-\widetilde{S}_{m m} \widetilde{g} .
$$

Then, in short hand, equation (3.11) becomes

$$
F\left(z_{s}\right)=h
$$

Note that the only terms entering into $F$ that do not depend on $z_{s}$ are the Cauchy data $\widetilde{f}$ and $\widetilde{g}$. For all the other terms the Fréchet derivatives with respect to $z_{s}$ are needed and put together by the product rule to create the Fréchet derivative of $F$. (We refrain from writing down the rather lengthy explicit expression for the derivative of $F$ in terms of the derivatives of all the operators and functions entering the definition of F.)

Given an approximate solution $z_{s}$ of (3.13), we need to solve the linearized equation

$$
d F\left[z_{s} ; \zeta\right]=h-F\left(z_{s}\right)
$$

for $\zeta$ to update the approximation $z_{s}$ into $z_{s}+\zeta$. Since the linearized equation is ill-posed regularization is required, for this we suggest the classical Tikhonov regularization with $H^{2}$ penalization. For a detailed introduction to the topic of regularized Newton iterations we refer to $[2,8]$.

The Fréchet derivatives of the integral operators given by (3.8) and (3.9) with respect to the boundary are obtained by differentiating their kernels with respect to $z_{s}$. The derivatives of the single-layer operators 
are given by

$$
\begin{aligned}
d \widetilde{S}_{s s}\left[\psi, z_{s} ; \zeta\right](t) & =-\frac{1}{\pi} \int_{0}^{2 \pi} \frac{\left[z_{s}(t)-z_{s}(\tau)\right] \cdot[\zeta(t)-\zeta(\tau)]}{\left|z_{s}(t)-z_{s}(\tau)\right|^{2}} \psi(\tau) d \tau, \\
d \widetilde{S}_{s m}\left[\psi, z_{s} ; \zeta\right](t) & =\frac{1}{\pi} \int_{0}^{2 \pi} \frac{\left[z_{m}(t)-z_{s}(\tau)\right] \cdot \zeta(\tau)}{\left|z_{m}(t)-z_{s}(\tau)\right|^{2}} \psi(\tau) d \tau, \\
d \widetilde{S}_{m s}\left[\psi, z_{s} ; \zeta\right](t) & =-\frac{1}{\pi} \int_{0}^{2 \pi} \frac{\left[z_{s}(t)-z_{m}(\tau)\right] \cdot \zeta(t)}{\left|z_{s}(t)-z_{m}(\tau)\right|^{2}} \psi(\tau) d \tau,
\end{aligned}
$$

for $t \in[0,2 \pi]$. The kernel of $d \widetilde{S}_{s s}$ is continuous with diagonal values

$$
-\frac{z_{s}^{\prime}(t) \cdot \zeta^{\prime}(t)}{\pi\left|z_{c}^{\prime}(t)\right|^{2}}
$$

The derivatives of the double-layer potentials are slightly more involved and are of the form

$$
d \widetilde{K}_{j k}\left[\psi, z_{s} ; \zeta\right](t)=\frac{1}{\pi} \int_{0}^{2 \pi}\left[\frac{P_{j k}(t, \tau)}{\left|z_{k}(t)-z_{j}(\tau)\right|^{2}}+\frac{R_{j k}(t, \tau)}{\left|z_{k}(t)-z_{j}(\tau)\right|^{4}}\right] \psi(\tau) d \tau
$$

for $t \in[0,2 \pi]$ and $j, k=m, s$. The numerators are given by

$$
\begin{aligned}
P_{s s}(t, \tau) & =\left[\zeta^{\prime}(\tau)\right]^{\perp} \cdot\left[z_{s}(t)-z_{s}(\tau)\right]+\left[z_{s}^{\prime}(\tau)\right]^{\perp} \cdot[\zeta(t)-\zeta(\tau)], \\
P_{s m}(t, \tau) & =\left[\zeta^{\prime}(\tau)\right]^{\perp} \cdot\left[z_{m}(t)-z_{s}(\tau)\right]-\left[z_{s}^{\prime}(\tau)\right]^{\perp} \cdot \zeta(\tau), \\
P_{m s}(t, \tau) & =\left[z_{m}^{\prime}(\tau)\right]^{\perp} \cdot \zeta(t),
\end{aligned}
$$

and by

$$
\begin{aligned}
R_{s s}(t, \tau) & =-2\left[z_{s}^{\prime}(\tau)\right]^{\perp} \cdot\left[z_{s}(t)-z_{s}(\tau)\right]\left[z_{s}(t)-z_{s}(\tau)\right] \cdot[\zeta(t)-\zeta(\tau)] \\
R_{s m}(t, \tau) & =2\left[z_{s}^{\prime}(\tau)\right]^{\perp} \cdot\left[z_{m}(t)-z_{s}(\tau)\right]\left[z_{m}(t)-z_{s}(\tau)\right] \cdot \zeta(\tau), \\
R_{m s}(t, \tau) & =-2\left[z_{m}^{\prime}(\tau)\right]^{\perp} \cdot\left[z_{s}(t)-z_{m}(\tau)\right]\left[z_{s}(t)-z_{m}(\tau)\right] \cdot \zeta(t) .
\end{aligned}
$$

The kernel of the operator $d \widetilde{K}_{s s}$ is continuous with the diagonal values

$$
\frac{\left[z_{s}^{\prime}(t)\right]^{\perp} \cdot \zeta^{\prime \prime}(t)+\left[\zeta^{\prime}(t)\right]^{\perp} \cdot z_{s}^{\prime \prime}(t)}{2 \pi\left|z_{s}^{\prime}(t)\right|^{2}}-\frac{\left[z_{s}^{\prime}(t)\right]^{\perp} \cdot z_{s}^{\prime \prime}(t) z_{s}^{\prime}(t) \cdot \zeta^{\prime}(t)}{\pi\left|z_{s}^{\prime}(t)\right|^{4}} .
$$


In view of the definition (3.10), the Fréchet derivative of $\widetilde{A}$ is given by

$$
\begin{aligned}
d \widetilde{A}\left(\psi, z_{s} ; \zeta\right):= & \frac{d}{d t} d \widetilde{S}_{s s}\left[\frac{d}{d t}\left(\widetilde{K}_{s s}-I\right)^{-1} \psi, z_{s} ; \zeta\right] \\
& -\frac{d}{d t} \widetilde{S}_{s s} \frac{d}{d t}\left(\widetilde{K}_{s s}-I\right)^{-1} d \widetilde{K}_{s s}\left[\left(\widetilde{K}_{s s}-I\right)^{-1} \psi, z_{s} ; \zeta\right] .
\end{aligned}
$$

The derivatives of $\widetilde{u}_{0}$ and $\widetilde{v}_{0}$ are obvious from (3.12).

The Newton iterations for (3.13) are closely related to the Newton iterations for the boundary-to-data operator

$$
G:\left.\Gamma_{s} \longmapsto \frac{\partial u}{\partial \nu}\right|_{\Gamma_{m}}
$$

that maps $\Gamma_{s}$ to the normal derivative of the solution to (1.1) and (2.1). For a given $\Gamma_{s}$, we can consider (3.1) also as a linear integral equation for the unknown normal derivative $g$ in the direct problem. From this, we observe that the boundary to data operator $G$ satisfies

$$
W G\left(\Gamma_{s}\right)=r\left(f, u_{0}\right),
$$

where

$$
W:=S_{m m}+\frac{1}{2}\left[K_{s m}-S_{s m} A\right] S_{m s}
$$

and

$$
\begin{aligned}
r\left(f, u_{0}\right):=\frac{1}{2}\left[K_{s m}-S_{s m} A\right] & \left(K_{m s} f+S_{s s} \frac{\partial u_{0}}{\partial \nu}-\left.K_{s s} u_{0}\right|_{\Gamma_{s}}-\left.u_{0}\right|_{\Gamma_{s}}\right) \\
& +f+K_{m m} f+S_{s m}\left(\frac{\partial u_{0}}{\partial \nu}-\left.A u_{0}\right|_{\Gamma_{s}}\right) .
\end{aligned}
$$

Therefore, in our approach, instead of $G\left(\Gamma_{s}\right)=g$, we linearize $W G\left(\Gamma_{s}\right)=W g$. If, in a slight abuse of notation, we identify $G$ with its parameterized version, from

$$
d\{W G-W g\}\left[z_{s}, \zeta\right]=d W\left[G\left(z_{s}\right)-g ; \zeta\right]+W d G\left[z_{s}, \zeta\right]
$$

and [4, Corollary 3.1], we can conclude that, for star-like boundaries, the linearized equation (3.1) is injective at the exact solution.

As an advantage of our approach as compared to the Newton iterations for the boundary-to-data map $G$ we note that here we have explicit expressions available for the Fréchet derivatives entering into the derivative of $F$ in terms of boundary integral operators. Opposed 
to this, the Fréchet derivative of $G$ is characterized in [4, Theorem 2.1] by the solution of transmission boundary value problems.

Although both the approach of this paper and the shape reconstruction part of the method proposed by Hanke and Rundell [3] are Newton type iterations, they differ conceptually. The shape reconstruction in [3] is based on the reciprocity gap principle, i.e., on Green's integral theorem with trigonometric harmonics as test functions, whereas the current approach may be viewed as based on the reciprocity gap principle with fundamental solutions as test functions, in addition to the reformulation of the source problem as a non-local Robin problem.

4. Numerical examples. As proof of concept rather than a documentation of a fully developed code, in this final section we present some numerical reconstructions. For these examples, the Neumann data were obtained by the integral equations presented in Section 2. Since these are based on a potential approach whereas the integral equations in the inverse algorithm are based on Green's formula, committing an inverse crime is avoided.

In principle, the parameterization of the update $\zeta$ obtained from (3.13) is not unique. To cope with this ambiguity, and in view of the uniqueness result in [5], we use star-like parameterizations of the form

$$
z_{s}(t)=r(t)(\cos t, \sin t), \quad 0 \leq t \leq 2 \pi,
$$

with a non-negative function $r$ representing the radial distance of $\Gamma_{c}$ from the origin. Consequently, the perturbations are of the form

$$
\zeta(t)=q(t)(\cos t, \sin t), \quad 0 \leq t \leq 2 \pi,
$$

with a real function $q$. In the approximations, we assume $r$ and its update $q$ to have the form of a trigonometric polynomial of degree $J$. In all of our examples we chose $J=8$ for exact data and $J=6$ for noisy data.

The three shapes to be reconstructed are a peanut-shaped curve

$$
z_{c}(t)=0.5 \sqrt{\cos ^{2}+0.25 \sin ^{2} t}(\cos t, \sin t),
$$

an apple-shape curve

$$
z_{c}(t)=\frac{0.5+0.4 \cos t+0.1 \cos 2 t}{1+0.7 \cos t}(\cos t, \sin t)
$$



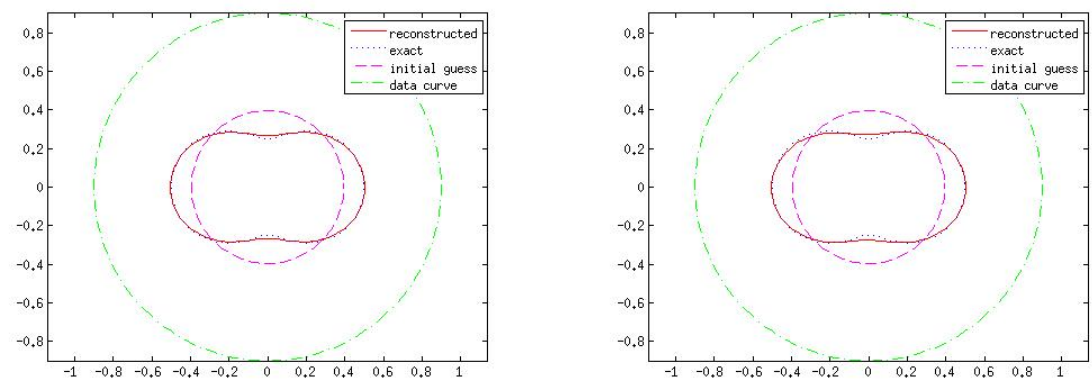

FiguRE 1. Reconstruction of (4.3) for exact data (left) and 3 percent noise (right).

and a kite-shaped curve

$$
z_{c}(t)=(-0.2+0.4 \cos t+0.2 \cos 2 t, 0.4 \sin t)
$$

for $0 \leq t \leq 2 \pi$. Note that none of the boundary curves belongs to the corresponding approximation space. The measurement curve $\Gamma_{m}$ is the circle with the representation

$$
z_{m}(t)=0.9(\cos t, \sin t), \quad t \in[0,2 \pi],
$$

where the factor 0.9 is chosen to satisfy the condition G. We used the Cauchy pair with Dirichlet data $f=0$.

In the figures the exact $\Gamma_{s}$ is given as dotted (blue) curve and the reconstruction as full (red) curve. The data curve $\Gamma_{m}$ is dashed-dotted (green) and the initial guess dashed (magenta).

For one iteration step, we collocated the linearized equation (3.1) at the points $t_{j}=j \pi / n, j=1, \ldots, 2 n$, and solved by Tikhonov regularization with an $H^{2}$ penalty term for the Fourier coefficients of the update trigonometric polynomials $q$. The number of collocation and quadrature points is $2 n=64$ on each curve.

The regularization parameter in the Tikhonov regularization was chosen by trial and error. However, to illustrate the feasibility and the stability of our method, we used the same regularization parameters in all examples. Depending on the $m$ th iteration step, for exact data the regularization parameters for an $H^{2}$ penalization was chosen as $\lambda=0.001 \times 0.8^{m}$ and for noisy data as $\lambda=0.01 \times 0.9^{m}$. For the 

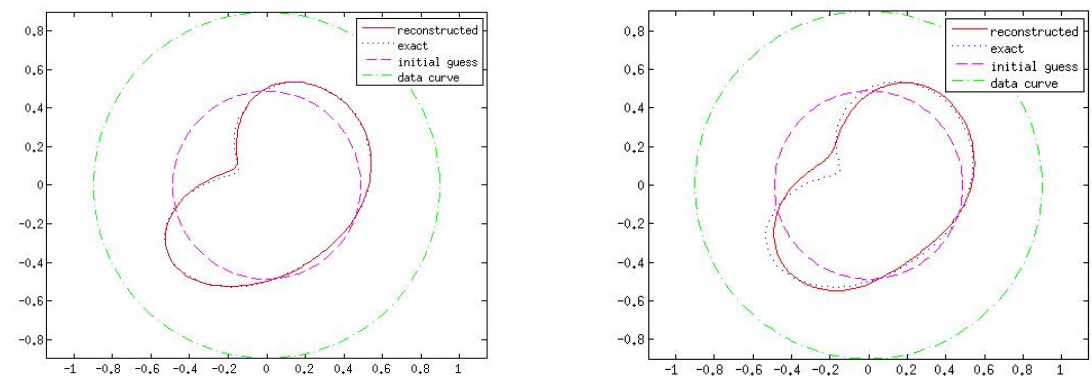

FiguRE 2. Reconstruction of (4.4) for exact data (left) and 3 percent noise (right).
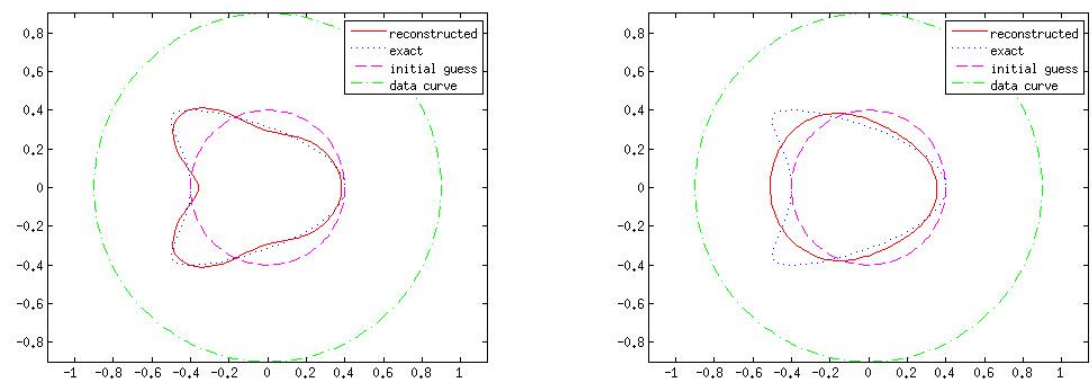

FiguRE 3. Reconstruction of (4.5) for exact data (left) and 3 percent noise (right).

perturbed data, random noise is added point-wise to the exact data, and the relative error is with respect to the $L^{2}$ norm.

As a stopping rule, we employed a type of discrepancy principle. For the exact data the iterations were stopped when the residual $\left\|F\left(z_{s}\right)-h\right\|_{L^{2}}$ became less than $0.5 \times 10^{-5}$ for the first time. This stopping number turned out to be $m=9$ for the peanut-shape, $m=18$ for the apple-shape and $m=29$ for the kite-shape. For noisy data, the iterations were stopped when the residual increased for the first time. Typically, this occurred between 5 and 12 iterations and, of course, depended on the individual perturbation.

The iterations were started with an initial guess given by a circle 
centered at the origin. When $\Gamma_{s}$ is a circle of radius $\rho$ from Green's integral theorem

$$
\int_{\Omega_{m}} \chi_{s} d x=\int_{\Gamma_{m}} g d s
$$

we have that

$$
\pi \rho^{2}=\int_{\Gamma_{m}} g d s .
$$

This suggests the use of (4.7) for the choice of the radius for the initial guess. However, we observed that the reconstruction algorithm is not too sensitive to the choice of the initial radius.

In principle, we also can shift the center of the star-like representation (4.1) by adding a constant vector $z_{0}$ to the representation of $z$. When $\Gamma_{s}$ is a circle of radius $\rho$, using Green's integral theorem, its center $z_{0}=z_{0,1}+i z_{0,2}$ can be obtained from

$$
\pi \rho^{2} z_{0}=\int_{\Gamma_{m}} w g d s,
$$

where $w\left(x_{1}, x_{2}\right)=z_{0}+x_{1}+i x_{2}$ for $\left(x_{1}, x_{2}\right) \in \mathbb{R}^{2}$. This suggests the use of (4.8) for the center $z_{0}$ in a shifted star-like representation. For an extension of our approach to multiple sources as indicated at the end of Section 1, the Padé algorithm in [3] generates centers for the subregions by a generalization of the use of (4.7) and (4.8).

\section{REFERENCES}

1. F. Cakoni, Y. Hu and R. Kress, Simultaneous reconstruction of shape and generalized impedance functions in electrostatic imaging, Inv. Prob. 30 (2014), 105009.

2. H.W. Engl, M. Hanke and A. Neubauer, Regularization of inverse problems, Kluwer Academic Publisher, Dordrecht, 1996.

3. M. Hanke and W. Rundell, On rational approximation methods for inverse source problems, Inv. Prob. Imag. 5 (2011), 185-202.

4. F. Hettlich and W. Rundell, Iterative methods for the reconstruction of an inverse potential problem, Inv. Prob. 12 (1996), 251-266.

5. V. Isakov, Inverse problems for partial differential equations, 2nd. edition, Springer, New York, 2005.

6. O. Ivanyshyn and R. Kress, Nonlinear integral equations for solving inverse boundary value problems for inclusions and cracks, J. Integral Equations Appl. 18 (2006), 13-38; Corrigendum: J. Integral Equations Appl. 22 (2010), 647-649. 
7. T. Johansson and B. Sleeman, Reconstruction of an acoustically sound-soft obstacle from one incident field and the far-field pattern, IMA J. Appl. Math. 72 (2007), 96-112.

8. B. Kaltenbacher, A. Neubauer and O. Scherzer, Iterative regularization methods for nonlinear ill-posed problems, de Gruyter, Berlin, 2008.

9. A. Kirsch, An introduction to the mathematical theory of inverse problems, 2nd. edition, Springer, New York, 2011.

10. R. Kress, Integral equations, 3rd. edition, Springer, New York, 2014.

11. , A collocation method for a hypersingular boundary integral equation via trigonometric differentiation, J. Integral Equations Appl. 26 (2014), 197-213.

12. R. Kress and W. Rundell, Nonlinear integral equations and the iterative solution for an inverse boundary value problem, Inv. Prob. 21 (2005), 1207-1223.

Institut für Numerische Und Angewandte Mathematik, Universität GöttinGen, D-37083 Göttingen, Germany

Email address: kress@math.uni-goettingen.de

Department of Mathematics, Texas A\&M University, College Station, TX 77843

Email address: rundell@math.tamu.edu 